\title{
Studies of one and two component aerosols using IR/VUV single particle mass spectrometry: Insights into the vaporization process and quantitative limitations
}

\author{
Emily A. Simpson, ${ }^{a}$ Pedro Campuzano-Jost, ${ }^{* a}$ Sarah J. Hanna, ${ }^{a}$ \\ Khalid M. M. Kanan, ${ }^{b}$ John W. Hepburn, ${ }^{a}$ Michael W. Blades ${ }^{a}$ and \\ Allan K. Bertram*a
}

Received 5th May 2010, Accepted 7th July 2010

DOI: $10.1039 / \mathrm{c0cp00462f}$

This paper presents the studies of one and two component particles using a $\mathrm{CO}_{2}$ laser for vaporization and VUV ionization in an ion trap mass spectrometer. The degree of fragmentation for a one component system was demonstrated to be a function of $\mathrm{CO}_{2}$ laser energy. In a two component system, the degree of fragmentation was shown to be a function of the particle composition. This observation indicates that the analysis of mixed particles may be far more complicated than anticipated for a two step process with soft vaporization. In addition to showing that fragmentation is a function of $\mathrm{CO}_{2}$ laser energy and particle composition, we also show that a key parameter that determines the extent of fragmentation is the energy absorbed by the particle during desorption. The ionization delay profile in a one component system is also shown to be strongly dependent on the vaporization energy. In a two component system, the delay profile is shown to strongly depend on the composition of the particle. The combined data suggest that the key parameter that governs the delay profile is the energy absorbed by the particle during desorption. This finding has implications for potential field measurements. Finally, for a two component system where the absorption crosssections are different, the change in the degree of fragmentation with particle composition resulted in a non-linear dependence of ion signal on composition. This makes any attempt at quantification difficult.

\section{Introduction}

Aerosols are an important topic of research given their ubiquitous presence in the atmosphere and their significant role in climate, chemistry of the atmosphere, and human health. ${ }^{1-4}$ Atmospheric aerosols contain numerous species, including sulfates, nitrates, and organics. One of the challenges in real-time organic aerosol analysis is the fragility of the molecules under study. One step laser desorption/ionization requires high laser powers which can cause extensive fragmentation of organic species through successive absorption of several photons and charge-transfer matrix effects. ${ }^{5}$ A solution to this is to separate the vaporization and ionization steps ${ }^{6}$ and use a "soft" ionization source to reduce fragmentation of many organics. ${ }^{7-9}$ Recently we have developed a single particle mass spectrometer that incorporates a $\mathrm{CO}_{2}$ laser for vaporization, a tunable laser-based VUV source for ionization, and an ion trap mass spectrometer for mass analysis. The instrument was characterized with single component particles by Hanna et al. ${ }^{8,10}$

By using a "soft" ionization source, ions are generated from the vaporized aerosol neutrals with a minimum of excess energy. This reduces the extent of fragmentation and thus simplifies the chemical characterization of the aerosol.

\footnotetext{
${ }^{a}$ Department of Chemistry, University of British Columbia, Vancouver, BC V6T 1Z1, Canada.

E-mail:pcampuzano@chem.ubc.ca,bertram@chem.ubc.ca

${ }^{b}$ Department of Chemistry, Al-Quds University, Jerusalem
}

To date, several research groups have implemented soft ionization sources for aerosol mass spectrometry. Some examples include metal attachment, ${ }^{7}$ chemical ionization, ${ }^{11-14}$ attachment of low-energy photoelectrons (PERCI), ${ }^{15-17}$ resonance enhanced multiphoton ionization (REMPI), ${ }^{6,18-24}$ and single photon ionization. ${ }^{9,25-31}$

In the following, we carry out detailed studies of pure oleic acid, pure oleyl alcohol (see Fig. 1 for chemical structures), and mixed oleic acid : oleyl alcohol particles using our single particle ion trap mass spectrometer. The three specific questions we are trying to address are detailed below.

Question 1: how does the fragmentation vary with $\mathrm{CO}_{2}$ energy and particle composition for one and two component systems? This is critical for interpreting mass spectra for similar instruments and also for determining if similar instruments will yield a linear response with particle composition. For example, if the fragmentation pattern of one species in a mixed particle changes as a function of the particle composition, evaluating the ion signal from a singular molecular or fragment ion would not give a linear response for that species.

Question 2: how does the ionization delay profile vary with $\mathrm{CO}_{2}$ energy and composition for one and two component systems? The ionization delay profile is a scan of total ion signal as a function of the delay time between the firing of the desorption laser and ionization laser. Understanding the ionization delay profiles is important for achieving optimal instrument performance, particularly in a field instrument where a single delay time will be used. 

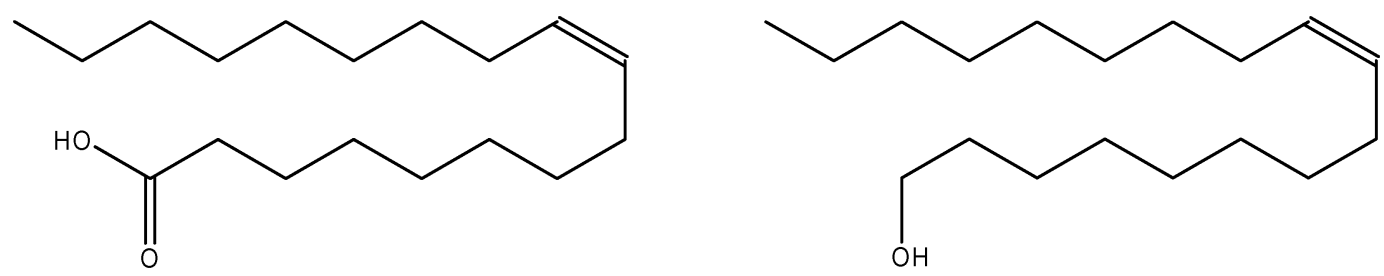

Fig. 1 Chemical structures of oleic acid (left) and oleyl alcohol (right).

Question 3: for a two component system in which the absorption crosssections are different, does the mass spectrum show a linear response with composition? The results from this study will help determine the usefulness of similar instruments for future quantitative and kinetic studies. Our results should apply to similar instruments that use a two step vaporization/ ionization process with a $\mathrm{CO}_{2}$ laser for desorption. This point is discussed in more detail in the Summary and conclusions section. Furthermore, this study will help identify some of the potential weaknesses of a two laser system for quantitative analysis of multi-component particles.

Oleic acid is present in the atmosphere at concentrations of $\sim 1 \mathrm{ng} \mathrm{m}^{-3}$ in the particle phase and is known to originate from a variety of sources, including meat cooking operations. ${ }^{32}$ Oleyl alcohol is structurally comparable to oleic acid with the carboxylic acid group being replaced by an alcohol (see Fig. 1). The two compounds are liquids and miscible in each other. Additionally, as the absorption crosssections of the 2 species at $1056 \mathrm{~cm}^{-1}$ (the $\mathrm{CO}_{2}$ wavelength utilized) differed by a factor of $\sim 4$ (based on the IR spectra measured; see Table 1), the instrument's ability to carry out quantitative measurements of mixtures of this kind is investigated.

There have not been many studies related to the questions outlined above. Studies of the effects of $\mathrm{CO}_{2}$ power on fragmentation have been previously conducted for single particles of oleic acid, ${ }^{31,33}$ ethylene glycol, ${ }^{28,31}$ and aniline ${ }^{5}$ with $\mathrm{CO}_{2} / \mathrm{VUV}$ in a TOF-MS. Hanna et al. investigated the same effect for oleic acid, 2,4-dihydroxybenzoic acid, and caffeine in an ion trap using a $\mathrm{CO}_{2}$ /VUV combination. ${ }^{8,10}$ There have been no previous studies of the change in fragmentation as a function of composition for mixed particles.

Studies of the ionization delay profile as a function of $\mathrm{CO}_{2}$ energy and/or composition have been done for single component particles of ethylene glycol, ${ }^{28}$ aniline, ${ }^{5}$ oleic acid, ${ }^{8}$ and $2,4-$ dihydroxybenzoic acid. ${ }^{8}$ In addition, Woods et al. ${ }^{30}$ discussed ionization delay profiles of mixed glycerol-oleic acid particles

Table 1 Relevant properties of oleic acid and oleyl alcohol

\begin{tabular}{lllll}
\hline \multicolumn{1}{l}{$\begin{array}{l}\mathrm{MW} / \mathrm{g} \\
\mathrm{mol}^{-1}\end{array}$} & $\begin{array}{l}\text { Density/g } \\
\mathrm{cm}^{-3}\end{array}$ & $\begin{array}{l}\sigma_{\mathrm{CO}_{2} / \mathrm{cm}^{2}} \\
\mathrm{molec}^{-1}\end{array}$ & $\mathrm{IE} / \mathrm{eV}$ \\
\hline Oleic acid & 282.4614 & 0.895 & $2.85 \times 10^{-20}$ & $\begin{array}{l}8.68^{27} \\
8.65 \pm 0.05^{8}\end{array}$ \\
$\begin{array}{l}\text { Oleyl } \\
\text { alcohol }\end{array}$ & 268.4778 & 0.850 & $1.03 \times 10^{-19}$ & $\begin{array}{l}<9.14^{a} \\
\end{array}$ \\
& & & $8.56 \pm 0.05^{b}$
\end{tabular}

${ }^{a}$ Based on energies given for 2-propen-1-ol and 2-butene-1-ol from NIST Chemistry Webbook and trends shown in Adam and Zimmermann ${ }^{37}$ for ionization energy as a function of increasing hydrocarbon chain length. ${ }^{b}$ Measured in a separate study in our group. (immiscible mixtures) using a $\mathrm{CO}_{2} / \mathrm{VUV}$ system. To our knowledge, no one has explored the effect of changing particle composition on ionization delay profiles (or noted such an effect) in a miscible mixed particle system.

Lastly, there has only been one study investigating the linear response with particle composition for mixed particles using $\mathrm{CO}_{2} /$ VUV. Baer and coworkers explored the quantitative use of $\mathrm{CO}_{2} / \mathrm{VUV}$ for four varying particle compositions of a three component polycyclic aromatic hydrocarbon $(\mathrm{PAH})$ mixture. ${ }^{29}$ These aromatic species are reasonably stable under fragmentation and primarily produce molecular ions which makes the overall analysis more straightforward. Conversely oleic acid and oleyl alcohol are not as robust as PAHs and tend to undergo more fragmentation, ${ }^{27,33}$ particularly in the ion trap system where storage time is longer $^{8}$ (see below for further discussion).

The two component system was studied at two $\mathrm{CO}_{2}$ desorption energies of $10 \mathrm{~mJ}$ per pulse and $7 \mathrm{~mJ}$ per pulse $\left(1056 \mathrm{~cm}^{-1}\right)$. At $10 \mathrm{~mJ}$ per pulse, both species fragmented extensively and it was impossible to separate the mass spectral peaks of the individual components; hence this paper focuses on results from the $7 \mathrm{~mJ}$ per pulse case.

\section{Materials and method}

\section{Instrument description}

The instrument has been described in detail in previous publications $^{8,10,34}$ and therefore only a brief description is given here. The main components of the system are an aerosol inlet, a sizing region, and a particle analysis region where aerosols are vaporized by a pulsed $\mathrm{CO}_{2}$ laser, the gas phase molecules ionized by pulsed vacuum ultraviolet (VUV), and the ions mass analyzed by an ion trap mass spectrometer (Fig. 2).

The mid-IR laser for desorption is a single mode, tunable pulsed $\mathrm{CO}_{2}$ laser $(9.2-10.8 \mu \mathrm{m})$ with a maximum output of $50 \mathrm{~mJ}$ on the strongest lines (MTL-3G, Edinburgh Instruments Ltd). In these studies, IR pulses with energies between 4-30 mJ were used to vaporize the particles with a laser spot size of $\sim 1 \mathrm{~mm}$ diameter inside the trap. For all the studies discussed in this paper a wavelength of $1056 \mathrm{~cm}^{-1}$ was used.

Particles are optically thin with respect to the IR energy in these experiments and are therefore expected to be uniformly heated by the $\mathrm{CO}_{2}$ laser pulse. A particle can be defined as optically thin if the product of the radius, $r$, the absorption crosssection, $\sigma$, and the concentration, $C$, is $\ll 1 .^{35}$ The IR absorption crosssections of oleic acid and oleyl alcohol (solutions prepared in dichloromethane) were measured using a Bruker Equinox 55 FTIR. At $1056 \mathrm{~cm}^{-1}$, the absorption crosssections were measured to be $2.85 \times 10^{-20} \mathrm{~cm}^{2}$ molec 


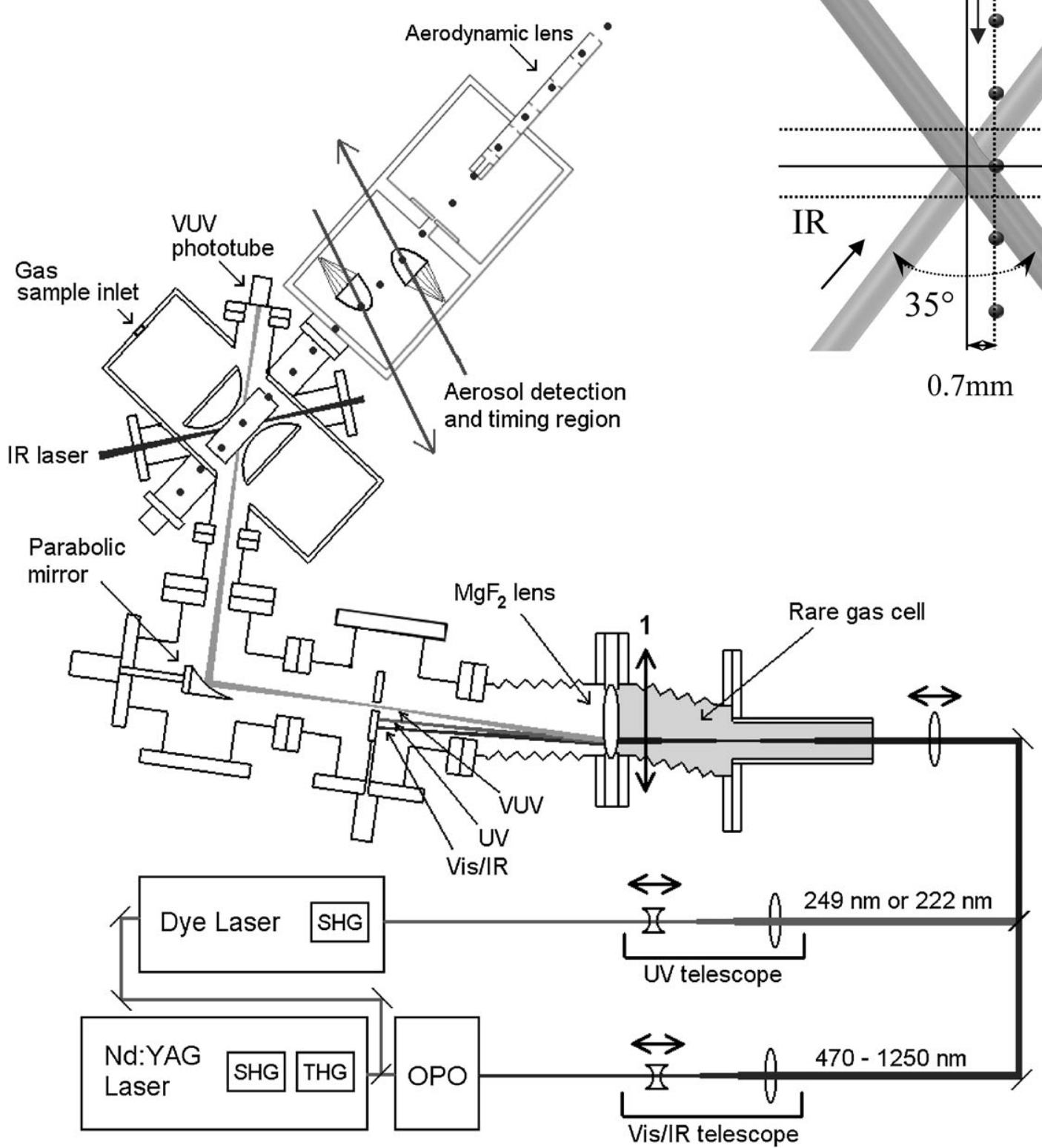

Fig. 2 Instrument schematic of the single particle ion trap mass spectrometer (SPITMS).

and $1.03 \times 10^{-19} \mathrm{~cm}^{2} \mathrm{molec}^{-1}$, respectively, for oleic acid and oleyl alcohol. Based on these numbers, $r \sigma C \ll 1$ for the experimental conditions. Additionally, the particle size is small in comparison to the $\mathrm{CO}_{2}$ wavelength making it unlikely that internal focusing of IR light will give rise to a temperature gradient in the particle. ${ }^{36}$

The tunable vacuum UV light is produced by resonance enhanced four wave difference mixing in xenon gas and is described in detail elsewhere. ${ }^{8,10}$ The source is continuously tunable from $10.2 \mathrm{eV}(122 \mathrm{~nm})$ to $7.4 \mathrm{eV}(168 \mathrm{~nm})$ and produces between $10^{10}$ and $10^{13}$ photons per pulse depending on the wavelength. The generated VUV is separated from the pump wavelengths by a custom monochromator and focused to a slightly vertically elongated spot with an area of $\sim 1 \mathrm{~mm}^{2}$.
For all the current studies an ionization energy of $8.75 \mathrm{eV}$ was used. For comparison, the measured ionization energy of oleic acid is $8.68 \mathrm{eV} .^{27}$ See Table 1 for some select properties of oleic acid and oleyl alcohol.

The ion trap is operated in mass selective instability mode with an accessible mass range of 10 to $340 \mathrm{Da}$. Mass scanning rates of $4000 \mathrm{Da} \mathrm{s}^{-1}$ were used for all the experiments which gives a mass resolution of $\sim 500 \mathrm{~m} / \Delta \mathrm{m}$ at $\mathrm{m} / \mathrm{z}=264$. The mass axis is calibrated daily by recording $70 \mathrm{eV}$ EI spectra of perfluorotributylamine.

It should be noted that the paths of the vaporization and ionization lasers and the path of the particle beam do not intersect in the center of the trap. Instead the particles first pass through the IR and then the VUV beam as they traverse 
the ion trap (inset of Fig. 2). The distance that the aerosols travel between the two intersection points is on the order of $2 \mathrm{~mm}$. As a result of this distance, there is no ion signal if the ionization pulse is fired immediately after the $\mathrm{CO}_{2}$ laser ( $0 \mu$ s delay time). However, if the delay between the two laser pulses is varied, a profile of the expanding plume from the vaporized aerosol can be obtained.

Experiments consisted of collecting mass spectra as a function of the delay time between the two laser pulses. Typically we scanned from $0-30 \mu$ s delay time. From these data we extracted the ionization delay profiles (scan of total ion signal as a function of time between desorption and ionization). See Fig. 5 as an example. The mass spectra presented in this paper were obtained by integrating over the entire delay profile. In other words, we summed all the mass spectra at each different delay time to obtain a representative mass spectrum for the entire vaporization process.

For the exploration of effects on fragmentation ratios, the integrated values over the entire delay profile were also used, again to give a fragmentation ratio consistent with the entire vaporization process.

\section{Chemicals}

Oleic acid (Fluka, $\geq 99 \%$ ) and oleyl alcohol (Aldrich, 99\%) were used as purchased without further purification. For pure particles, solutions were prepared in 2-propanol (Aldrich, 99.9\%) in the concentration range of $10^{-5} \mathrm{~g} \mathrm{~mL}^{-1}$ and particles were generated using a vibrating orifice aerosol generator (TSI Model 3450).

Mixed particles of oleic acid and oleyl alcohol were prepared by aerosolizing solutions with mass ratios of $90: 10,75: 25,50: 50$, and $25: 75$ oleic acid : oleyl alcohol in isopropanol with the total mass concentration of the solution remaining unchanged $\left(2 \times 10^{-5} \mathrm{~g} \mathrm{~mL}^{-1}\right)$. Since the molar masses of oleic acid and oleyl alcohol are very similar, the mole ratios vary from the mass ratios by at most $1 \%$.

All particles were passed through a ${ }^{85} \mathrm{Kr}$ charge neutralizer with $\sim 25$ LPM dilution air flow before entering the single particle ion trap mass spectrometer (SPITMS). This acted as a drying tube and prevented additional drying and size changes in the aerosol lens.

\section{Results}

\section{Pure oleic acid particles}

Particle mass spectra were acquired for pure one component particles of oleic acid using a range of $\mathrm{CO}_{2}$ energies for vaporization. Fig. 3 shows an example spectrum recorded at $7 \mathrm{~mJ}$ per pulse $\mathrm{CO}_{2}$ energy. No ions from the $\mathrm{CO}_{2}$ laser alone were observed over the entire range of energies. The mass spectra showed a shift towards lower $m / z$ fragments with increasing vaporization energy as expected. ${ }^{31}$

Several peaks from the oleic acid mass spectrum were used to assess the degree of fragmentation as a function of $\mathrm{CO}_{2}$ energy. The peaks chosen for this analysis were selected to cover a range of fragment ions from high $\mathrm{m} / \mathrm{z}$ to low $\mathrm{m} / \mathrm{z}$ across the spectrum. These peaks are labeled in Fig. 3. Each fragment's relative intensity is determined with respect to the

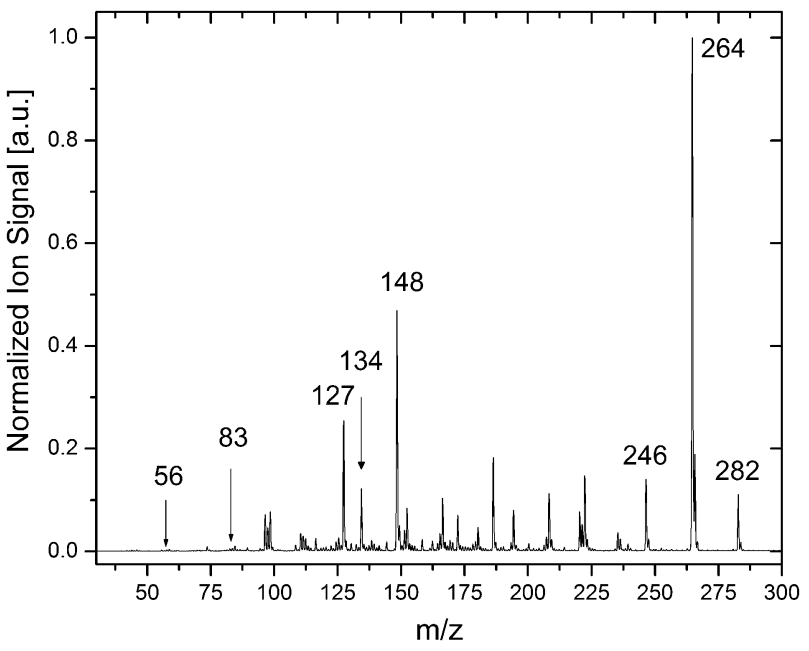

Fig. 3 Oleic acid normalized mass spectra (averaged over the entire delay profile) acquired at $7 \mathrm{~mJ} 1056 \mathrm{~cm}^{-1} \mathrm{CO}_{2}, 142 \mathrm{~nm}$ VUV.

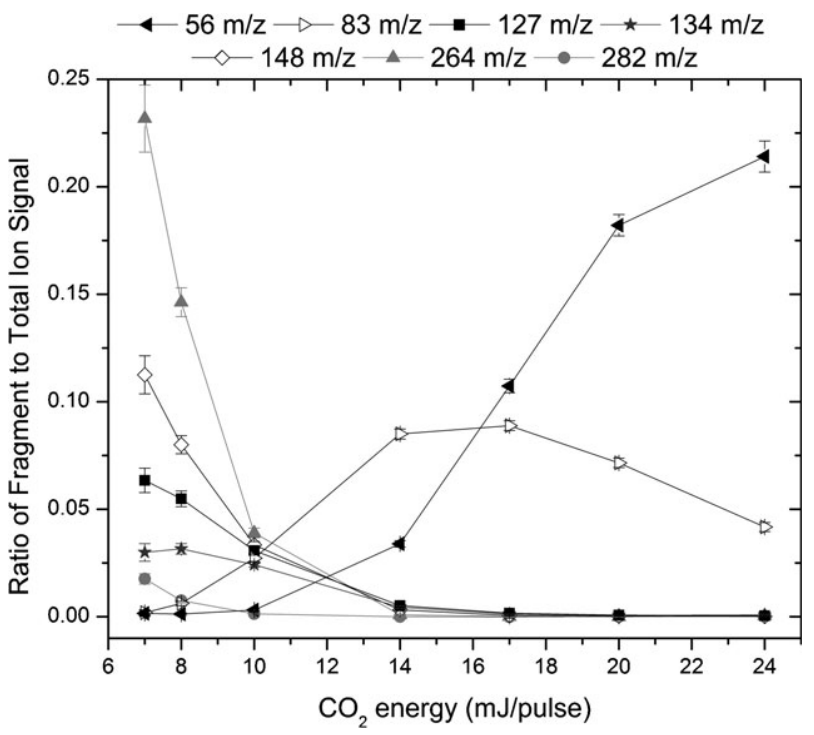

Fig. 4 Relative intensities of fragment peaks compared to the total ion signal as a function of desorption energy for pure oleic acid particles.

total ion signal. As can be seen in Fig. 4, the general trend is a decrease in high mass peaks and an increase in lower mass fragments with increasing desorption energy. This changing degree in fragmentation reflects the increasing internal energy of the vaporized molecules. ${ }^{28}$

Fig. 5 shows the ionization delay profiles obtained for oleic acid over a range of desorption energies. The exact shape of the profile is dependent on the distance between the two laser beams (which is constant in these experiments) and the translational energy of the vaporized molecules which expand outward from the particle, filling, and then passing beyond the ionization volume. As can be seen, the expansion speed of the desorbed aerosol plume increases with increasing $\mathrm{CO}_{2}$ laser energy, with the maximum total ion signal shifting to shorter delay times. These profiles were normalized for ease of comparing the optimal delay time where maximum ion signal 


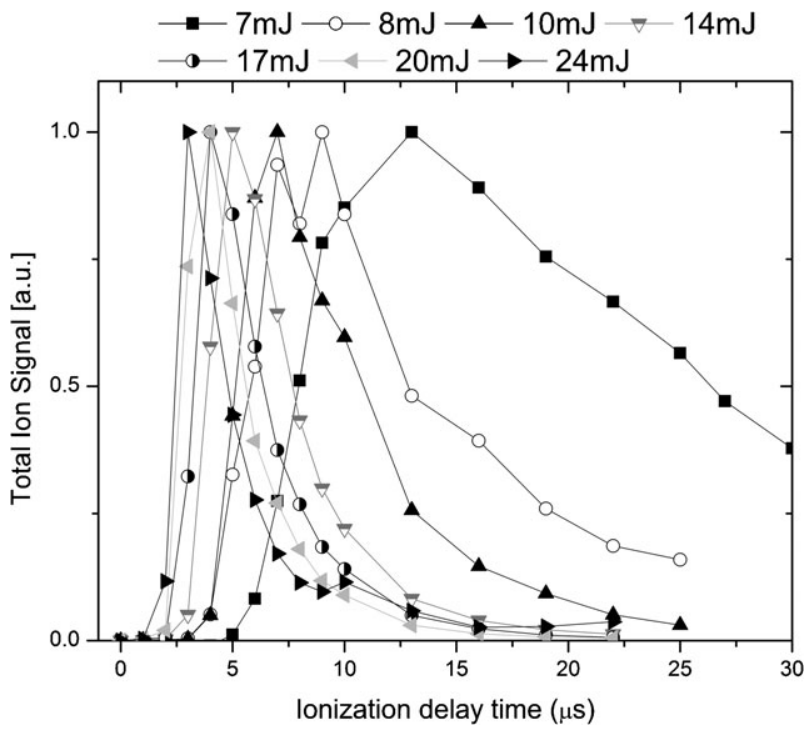

Fig. 5 Total ion signal as a function of ionization delay time for oleic acid. Each trace is for a different $\mathrm{CO}_{2}$ pulse energy $\left(\right.$ at $1056 \mathrm{~cm}^{-1}$ ) as indicated in the legend (VUV at $142 \mathrm{~nm}$ ). All traces have been normalized to make comparison easier.

occurs. It should be noted that total ion intensities do change with increasing desorption power, reaching a plateau in total ion signal at approximately $15 \mathrm{~mJ}$ per pulse. Also, note that the ionization delay profile is only a qualitative indicator of translational energy, since energy distributions from the vaporization process can be complex and are not well understood.

\section{Pure oleyl alcohol particles}

Particle mass spectra were also acquired for pure one component particles of oleyl alcohol using a range of $\mathrm{CO}_{2}$ energies. Fig. 6 shows an example spectrum recorded at $7 \mathrm{~mJ}$ per pulse $\mathrm{CO}_{2}$ energy. Increasing the desorption energy resulted in increasing levels of fragmentation in concurrence with previous studies. ${ }^{5,8,10,28,31,33}$ No molecular ion was observed at any of the desorption energies used and the fragmentation was quite extensive even at the lowest vaporization energy used.

The peaks chosen for this analysis were selected to cover a range of fragment ions from high $\mathrm{m} / z$ to low $\mathrm{m} / z$ across the spectrum. The intensities of selected fragment peaks in the mass spectrum (see Fig. 6) were again compared to the total ion signal as a function of desorption energy to give an indication of the degree of fragmentation for oleyl alcohol. Fig. 7 shows the same trend observed for oleic acid: a decrease in high mass fragments and increase in low mass fragments as desorption energy increases.

Fig. 8 shows the normalized ionization delay profiles obtained for oleyl alcohol at a range of desorption energies. These profiles were normalized for ease of comparing the optimal delay time where maximum ion signal occurs. It should be noted that total ion intensities do change with increasing desorption power, reaching a plateau in total ion signal at approximately $10 \mathrm{~mJ}$ per pulse for oleyl alcohol. An increase in the expansion speed of the desorbed aerosol plume can be observed with increasing $\mathrm{CO}_{2}$ laser energy

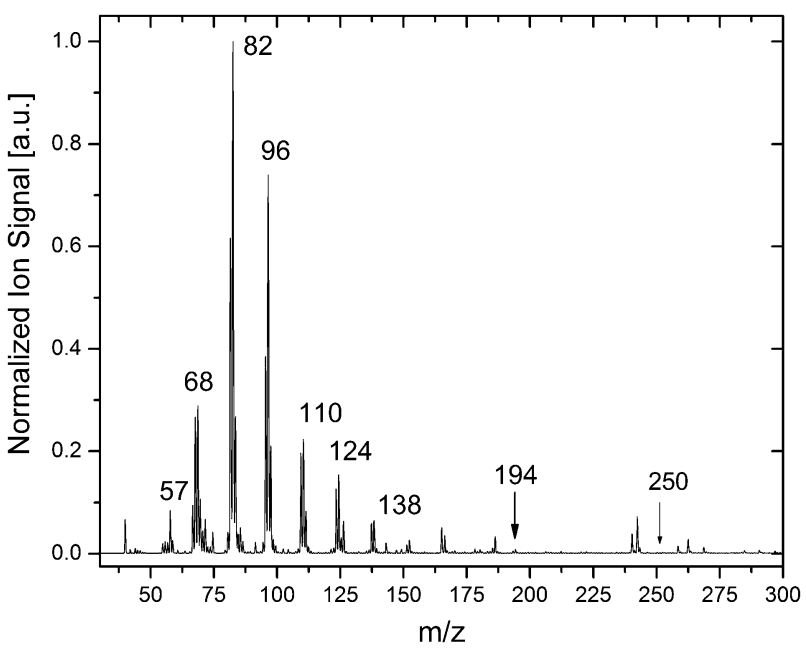

Fig. 6 Oleyl alcohol normalized mass spectra (averaged over the entire delay profile) acquired at $7 \mathrm{~mJ} 1056 \mathrm{~cm}^{-1} \mathrm{CO}_{2}, 142 \mathrm{~nm}$ VUV.

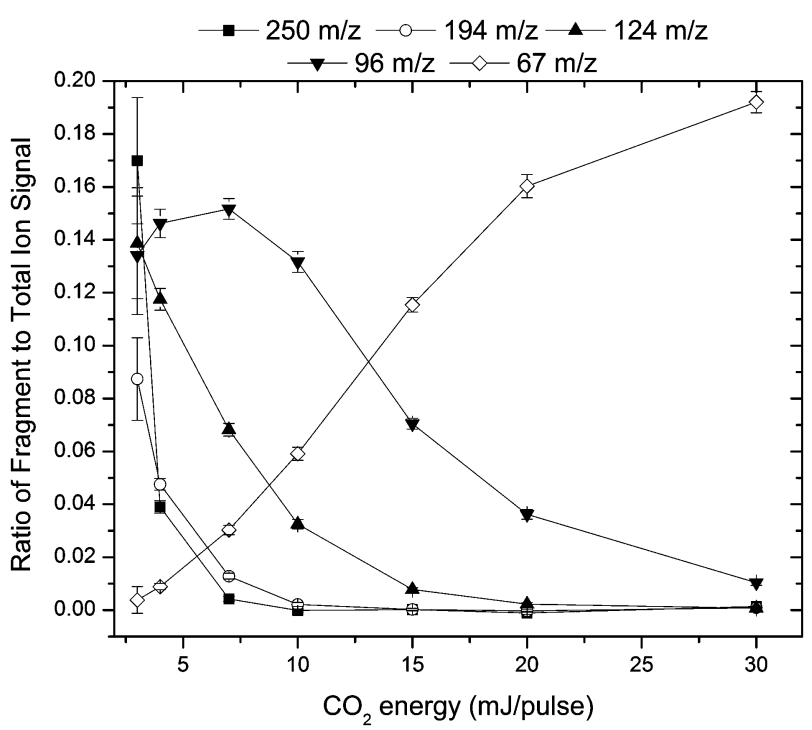

Fig. 7 Relative intensities of fragment peaks compared to the total ion signal as a function of desorption energy for pure oleyl alcohol particles.

similar to what was observed for pure oleic acid particles and in other studies. However, it is observed that at a comparable desorption energy, for instance $7 \mathrm{~mJ}$, there is a significant difference in the delay profiles observed for oleic acid and oleyl alcohol. For pure oleyl alcohol, the maximum ion signal occurs at $6 \mu$ s compared to $14 \mu$ s for pure oleic acid. The delay profile reaches a maximum ion signal at a shorter delay time and decays faster for oleyl alcohol, suggesting a much faster translational energy of desorbed molecules as compared to oleic acid. The differences in delay profiles are likely due to the differences in IR absorption crosssections of oleyl alcohol and oleic acid for an equivalent $\mathrm{CO}_{2}$ laser power. As mentioned previously, the absorption crosssection of oleyl alcohol was approximately a factor of 4 higher in magnitude than that of oleic acid based on the IR spectra of the pure components. This concept is explored in more detail in the Discussion section. 


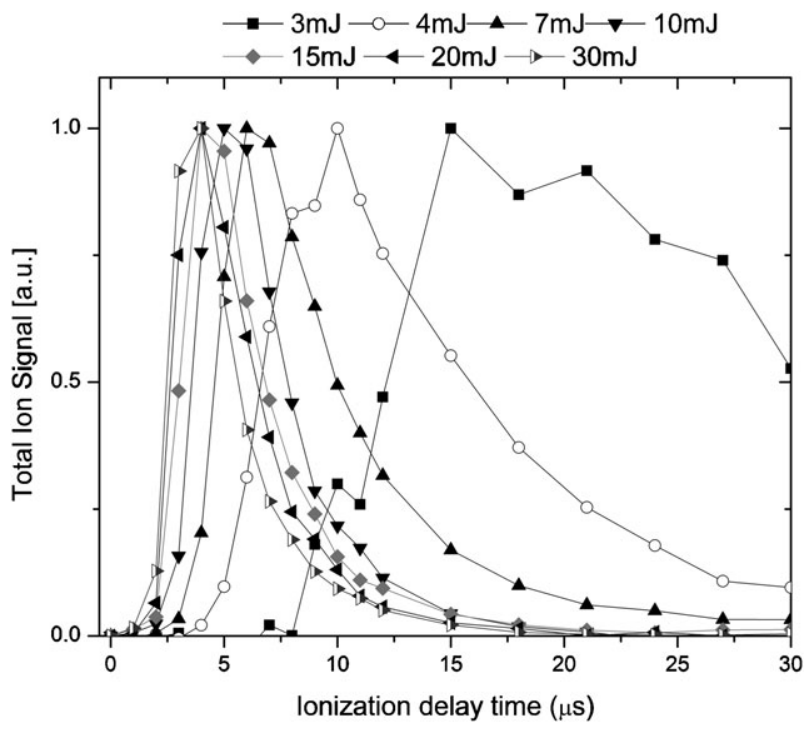

Fig. 8 Total ion signal as a function of ionization delay time for oleyl alcohol at an ionization wavelength of $142 \mathrm{~nm}$. Each trace is for a different $\mathrm{CO}_{2}$ pulse energy (at $1056 \mathrm{~cm}^{-1}$ ) as indicated in the legend. All traces have been normalized to make comparison easier.

\section{Two component particles of oleic acid and oleyl alcohol}

All results discussed for the two component particles were obtained with $7 \mathrm{~mJ}$ per pulse desorption energy, where the oleic acid mass spectrum has distinctive high mass peaks $(m / z=264,246,148,134,127)$ that do not overlap with oleyl alcohol fragments. Due to the fragmentation of metastable ions observed in the ion trap previously, ${ }^{8}$ we don't see significant molecular ion peaks for the analytes chosen in this study (Fig. 3 and 6). This adds to the complexity of analysis.

Normalized mass spectra are shown as a function of particle composition in Fig. 9. The pure particle spectra for oleic acid and oleyl alcohol are distinctly different at $7 \mathrm{~mJ} 1056 \mathrm{~cm}^{-1} \mathrm{CO}_{2}$. Oleic acid experiences far less fragmentation and produces fragments primarily above $m / z 125$, while oleyl alcohol undergoes much more fragmentation and gives fragments primarily below $m / z$ 125. The mass spectra of the mixed particles show some interesting features. The $90: 10$ wt $\%$ spectrum appears to most closely resemble a combination of the peak intensities found in each of the $7 \mathrm{~mJ}$ pure component spectra. By $75: 25 \mathrm{wt} \%$, the dominant peaks for oleic acid (at $m / z=264$ for example) are extremely diminished, more so than would be expected based on composition alone. This could be due to a shift in the fragmentation pattern of oleic acid, perhaps due to experiencing more heating in the vaporization step or it could be due to a preferential vaporization of oleyl alcohol. However, as the two components are both liquids and miscible, it is not expected that oleyl alcohol partitions to the surface and undergoes preferential desorption at low $\mathrm{CO}_{2}$ energies; therefore a change in the oleic acid fragmentation pattern seems most likely.

The fragmentation of the mixed particles was then examined as a function of composition. Since oleyl alcohol is more heavily fragmented at $7 \mathrm{~mJ}$, selected high mass peaks in oleic acid were used to monitor the change in fragmentation. Peaks at $m / z 127,134,235,246$, and 264 were ratioed to $m / z 148$ to

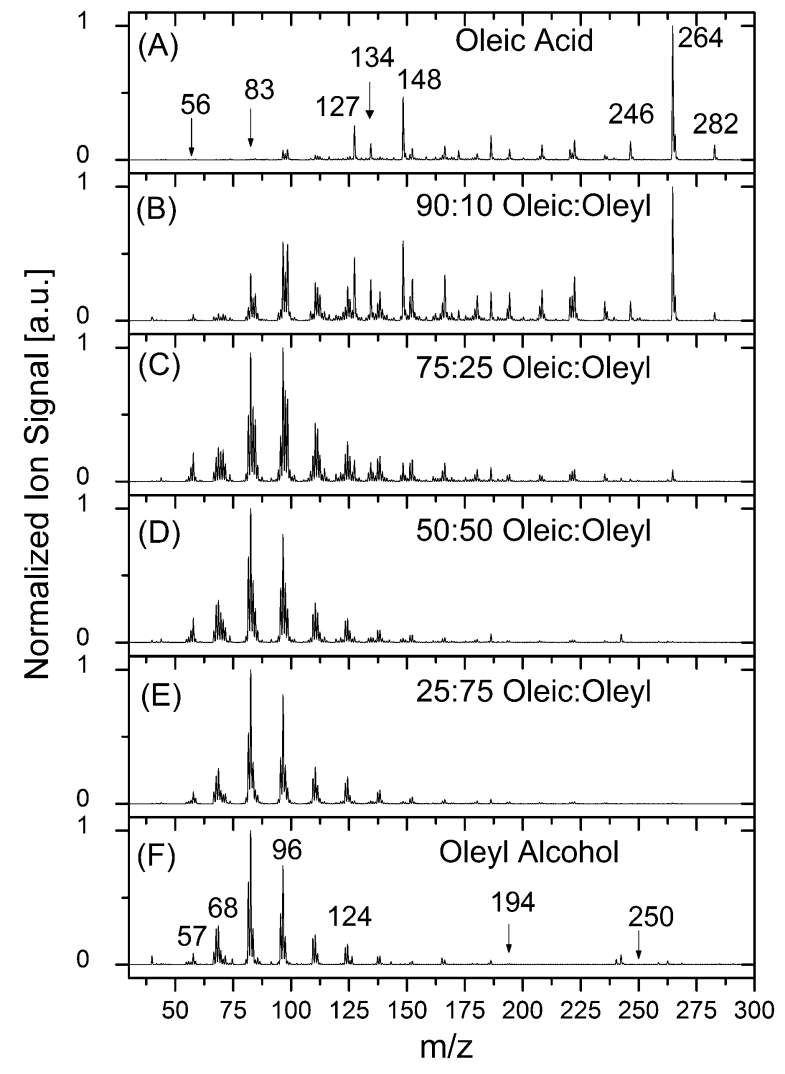

Fig. 9 Normalized mass spectra (averaged over the entire delay profile) as a function of particle composition $\left(7 \mathrm{~mJ} 1056 \mathrm{~cm}^{-1} \mathrm{CO}_{2}\right.$, $142 \mathrm{~nm}$ VUV).

determine the degree of fragmentation of oleic acid. All of these peaks are assigned exclusively to oleic acid. Oleyl alcohol spectra were examined in detail at a range of different $\mathrm{CO}_{2}$ energies to ensure that none of the peaks selected to evaluate oleic acid were observed. Therefore we can use these peaks with confidence to look at the change in fragmentation for oleic acid by comparing to the peak at $m / z$ 148, which also belongs exclusively to oleic acid and is present at a range of energies in the pure oleic acid spectra. All other peaks that we considered using for comparison had extensive overlap with oleyl alcohol fragments. For example, the oleic acid fragment at $56 \mathrm{~m} / z$ overlaps with an oleyl alcohol peak within the cluster of fragments around $57 \mathrm{~m} / \mathrm{z}$ that can be seen in Fig. 6 .

Fig. 10 shows the degree of fragmentation as a function of composition for oleic acid in mixed particles. The fragmentation pattern for oleic acid clearly shows that as the percentage composition of oleic acid decreases (oleyl alcohol increases), oleic acid fragments more extensively.

The ionization delay profiles (total ion signal versus ionization delay time) also reflect the effect of composition on vaporization of the particle. In Fig. 11, it can be seen that the ionization delay profiles change noticeably with composition until a certain percentage of oleyl alcohol is reached in the particle ( $\sim 25 \%$ or greater). There is also a significant change in total ion signal with an increasing amount of particle material desorbed with increasing oleyl alcohol composition which is not reflected by the normalized delay profiles. 


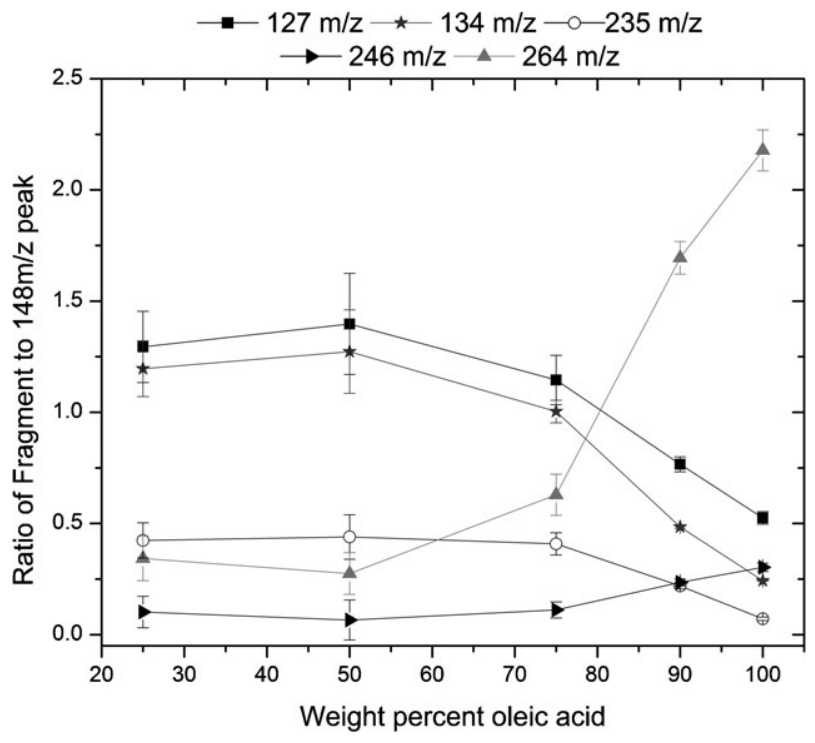

Fig. 10 Fragmentation ratio of oleic acid (selected peaks relative to $\mathrm{m} / \mathrm{z} 148)$ as a function of weight percent oleic acid.

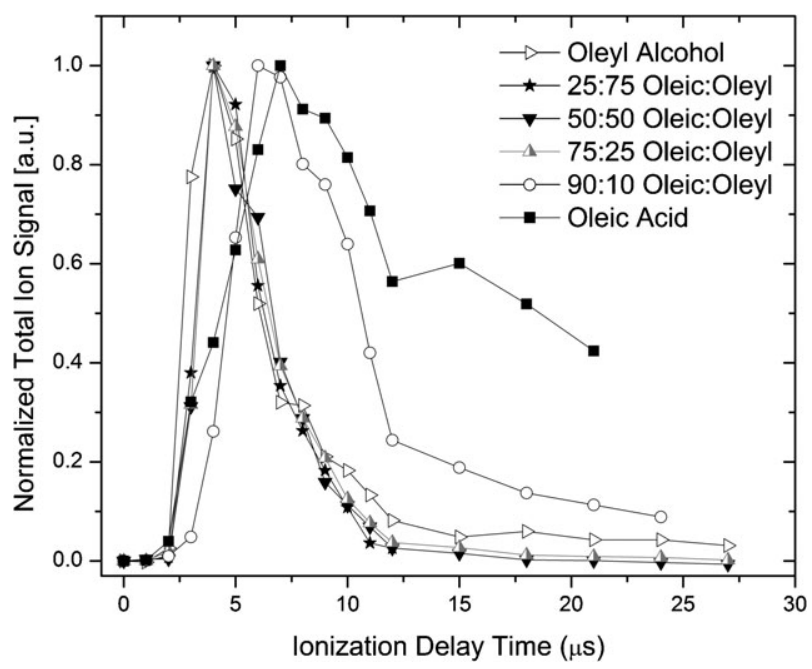

Fig. 11 Normalized ion signal as a function of ionization delay time for mixed oleic acid : oleyl alcohol particles.

\section{Discussion}

\section{Dependence of degree of fragmentation on $\mathrm{CO}_{2}$ energy and composition}

The degree of fragmentation for a species has been shown to be strongly related to the internal energy of the molecule. ${ }^{8,9,28,31,33,35,38,39}$ If the internal energy of the vaporized molecules is high, then increased fragmentation is expected..$^{35,38-40}$ For the pure particles in this study, fragmentation increased with increasing desorption energy, which is consistent with results from other groups that show the degree of fragmentation to be a strong function of particle heating, regardless of whether a heater or laser was used for the vaporization step. ${ }^{8,9,28,31,33,35,38,39}$ Additionally, in this study the extent of fragmentation was seen to depend on particle composition. At an equal desorption energy, pure oleyl alcohol showed more fragmentation than pure oleic acid.
The partitioning of energy into the different degrees of freedom is complex and not well understood in laser vaporization of particles. Here we only carry out a simple qualitative analysis to show that the fragmentation behavior observed for pure particles as well as the two component particles comes from the different amounts of absorbed $\mathrm{CO}_{2}$ energy. In other words, this simple analysis shows that the presence of oleyl alcohol in the mixed particle enhances the absorption of $\mathrm{CO}_{2}$ laser radiation and leads to an increase in internal energy (and hence fragmentation) of the vaporized molecules.

The respective absorption crosssections determined in the IR measurements were used to calculate the amount of energy absorbed by the particle $\left(E_{\mathrm{abs}}\right)$ for both the pure oleic acid particles and the mixed particles according to Beer's law and some simple approximations as described by eqn (1) and (2). The derivation of eqn (1) (for a one component system) is given in the Appendix.

$$
E_{\mathrm{abs}}=E_{\mathrm{CO}_{2}} \frac{A_{\mathrm{p}}}{A_{\mathrm{CO}_{2}}}\left\{1-\exp \left[-\sigma N_{\mathrm{p}}\left(\frac{4 r_{\mathrm{p}}}{3}\right)\right]\right\}
$$

$E_{\mathrm{CO}_{2}}$ is the measured $\mathrm{CO}_{2}$ energy, $A_{\mathrm{p}}$ is the area of the particle, $A_{\mathrm{CO}_{2}}$ is the area of the $\mathrm{CO}_{2}$ beam where it intersects the particle, $\sigma$ is the absorption crosssection, $N_{\mathrm{p}}$ is the number density of molecules in the particle, and $4 r_{\mathrm{p}} / 3$ is an approximation of the path length the light travels through the particle. Similarly for a two component system, the equation for energy absorbed by the particle during desorption is given below:

$$
E_{\text {abs }}=E_{\mathrm{CO}_{2}} \frac{A_{\mathrm{p}}}{A_{\mathrm{CO}_{2}}}\left\{1-\exp \left[-\left(\sigma_{1} N_{1}+\sigma_{2} N_{2}\right) \frac{4 r_{\mathrm{p}}}{3}\right]\right\}
$$

where $\sigma_{1}$ and $\sigma_{2}$ are the respective crosssections of each component and $N_{1}$ and $N_{2}$ are the respective number densities of each component in the mixed particle.

Fig. 12 shows the ratios of the peak intensities at $m / z=127$, 134 and 264 relative to the peak at $m / z 148$ as a function of the calculated $\mathrm{CO}_{2}$ energy absorbed per particle for oleic acid in both the one component and two component particles. The closed data points represent the pure oleic acid particles and the open data points represent the mixed particles. The ratio of $m / z=264$ to $m / z=148$ decreases with increasing energy absorbed whereas the ratio of peaks at $m / z 127$ and 134 to $m / z 148$ increases with increasing energy absorbed. At higher energies for the pure oleic acid, the error bars are large due to the low signal to noise ratio which occurs because the mass spectra are dominated by very low mass fragments $(<m / z=50)$ at these energies. The data in Fig. 12 demonstrate that the degree of fragmentation for oleic acid in both one component and two component particles is due to the energy absorbed per particle in the desorption step. As expected, more energy absorbed results in more fragmentation.

\section{Dependence of ionization delay profiles on $\mathrm{CO}_{2}$ laser energy and particle composition}

The expansion of the aerosol plume can be followed by varying the delay between the $\mathrm{CO}_{2}$ and VUV pulses. The shape of the ionization delay profile as illustrated by the profile FWHM or the delay time at maximum ion signal is a qualitative indicator of the translational energy of the 


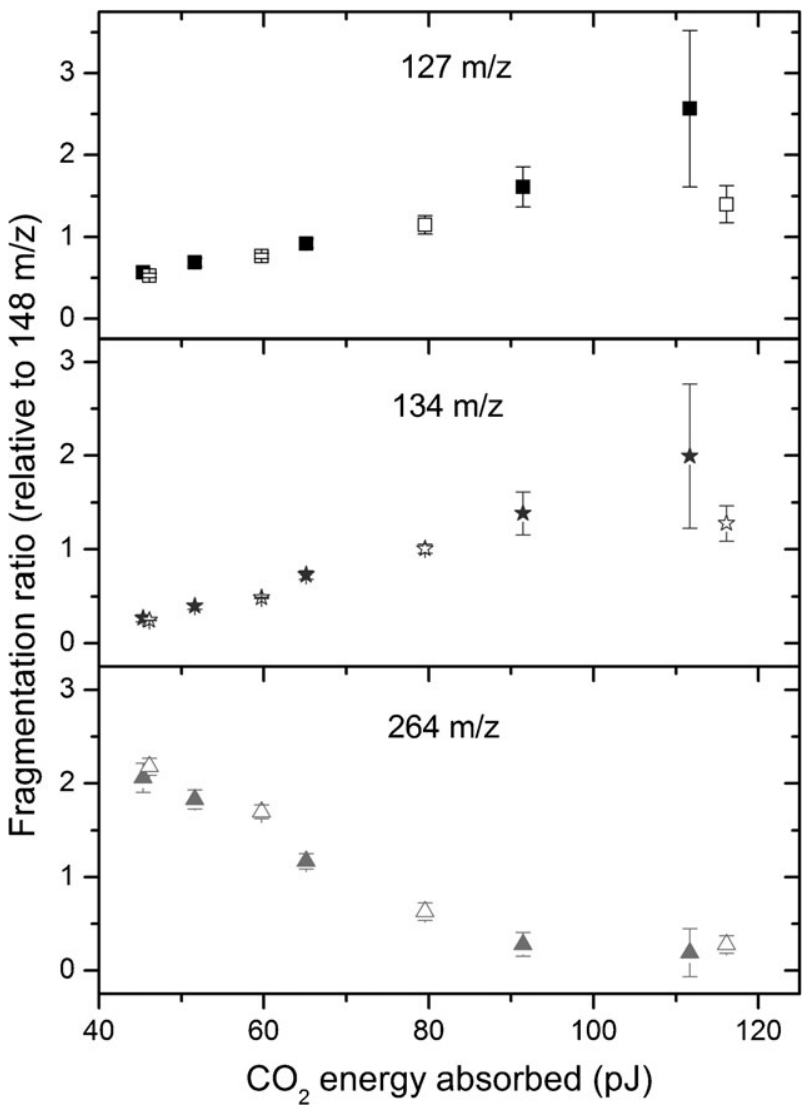

Fig. 12 Fragmentation ratios for oleic acid as a function of IR energy absorbed during vaporization. Closed symbols show pure oleic acid particle data, open symbols show mixed particle data.

vaporized molecules. ${ }^{28}$ The pure component data suggest that the relative translational energy of the desorbed molecules increased with increasing $\mathrm{CO}_{2}$ energy leading to narrower delay profiles and earlier optimal delay times. In the case where a higher translational energy is imparted to the molecules, they will enter and leave the ionization region more quickly, whereas at lower energies the molecules will take longer to fill and then expand beyond the ionization volume. In the mixed particle study, all particles are vaporized at the same $\mathrm{CO}_{2}$ laser energy of $7 \mathrm{~mJ}$, yet the relative translational energy of the molecules appears to be changing with composition as shown in Fig. 10.

Similar to the previous section, we perform a simple qualitative analysis to show that the trends in the ionization delay profiles for both the one component and two component aerosols come from the different amounts of absorbed $\mathrm{CO}_{2}$ energy. Fig. 13 contains the pure and mixed particle data for oleic acid plotted as ionization delay profile FWHM (full width at half maximum) versus absorbed energy in the top panel and optimum delay time versus absorbed energy in the bottom panel. Closed data points represent the pure particle and open data points represent the two component particle. Both panels demonstrate a fairly consistent trend: the FWHM and delay time both decrease with an increase in $\mathrm{CO}_{2}$ energy absorbed. Overall, Fig. 13 illustrates that more absorbed $\mathrm{CO}_{2}$ energy results in a higher relative translational energy, as expected.

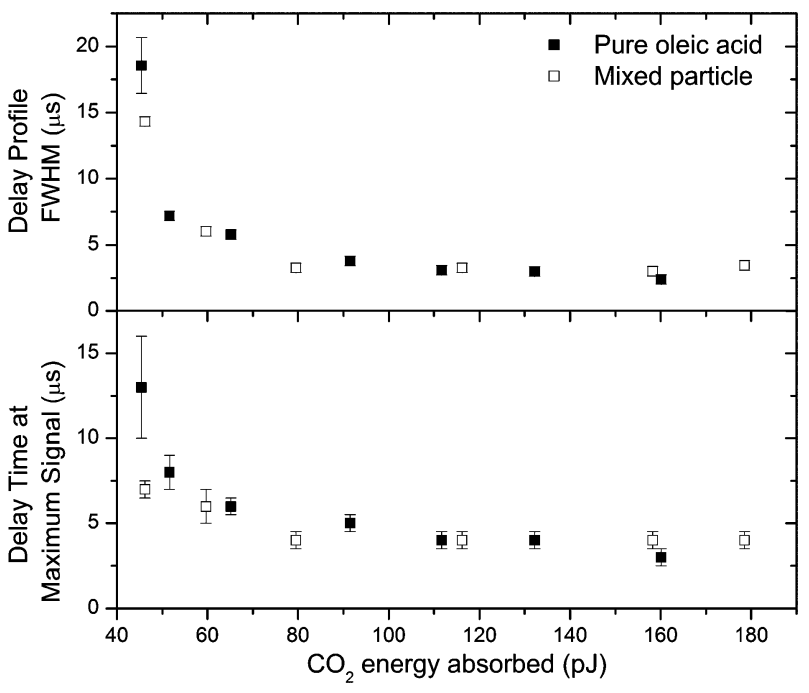

Fig. 13 (Top) Ionization delay profile FWHM for both pure oleic acid particles and mixed particles as a function of $\mathrm{CO}_{2}$ energy absorbed/particle and (bottom) delay time at maximum total ion signal as a function of $\mathrm{CO}_{2}$ energy absorbed/particle.

\section{Non-linear response of ion signal with particle composition}

In a previous study, Woods et al. showed that a two step process using VUV ionization gave quantitative detection of aromatics (PAHs) in a mixed particle. ${ }^{29}$ In other words, the signal from a single component in a mixed particle was linear with the amount (mole fraction) of that component in the mixed particle. Shown in Fig. 14 is a plot of three peaks (assigned exclusively to oleic acid) normalized to the total ion signal as a function of concentration. By normalizing to the total ion signal any effect of partial or incomplete vaporization on the quantitative response should be removed. The spectra were collected from $30-300 \mathrm{~m} / z$ and it is not believed that any significant amount of fragments occurs below $30 \mathrm{~m} / \mathrm{z}$ based on previously collected data. Therefore, the relative amount of oleic acid fragments should give a linear response in relation to composition even if the amount of material evaporating

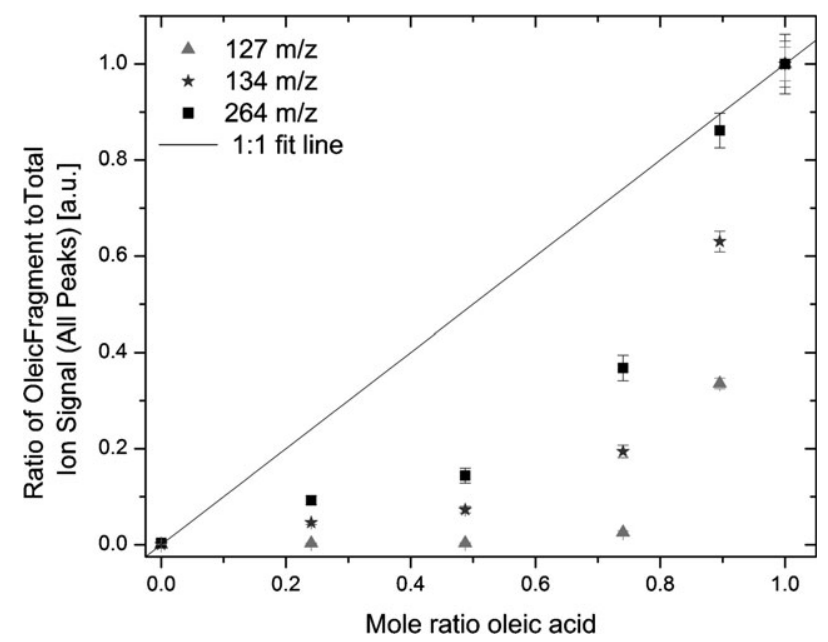

Fig. 14 Relative intensity of oleic acid fragment ion signal to total ion signal for all peaks (oleic and oleyl) as a function of the oleic acid mole ratio. 
changes as a function of composition. However, the trend shown in Fig. 14 is clearly non-linear over the range of compositions. This trend most likely occurs because the relative amount of signal at $m / z 127,134$ and 264 is changing due to: (1) a change in composition and (2) a change in fragmentation with composition. If every peak in the mass spectrum from oleic acid could be included in the analysis, a linear trend would be expected. Conversely, this would be very difficult due to the issue with overlapping peaks between similar aliphatic species. To illustrate the problem with overlapping peaks, in our experiments only peaks at $m / z 282,264$, $246,148,134$, and 127 can be exclusively assigned to oleic acid. All other peaks overlap with oleyl alcohol peaks. For a $50: 50$ mixture of oleic acid and oleyl alcohol, these peaks assigned exclusively to oleic acid contribute $<5 \%$ to the total ion signal.

Fig. 14 shows that a quantitative analysis is difficult if the energy absorbed per particle during desorption changes significantly with composition. Conditions where quantitative analysis should be successful are (1) where the fragmentation does not change with composition or (2) where the IR absorption crosssections do not change with composition (assuming heat capacities are similar). An example of the first case would be analytes that do not fragment or produce relatively few fragments, such as the mixtures of PAHs studied by Woods et al. ${ }^{29}$ For the second case, species with similar absorption crosssections and heat capacities are needed or the change in composition restricted to a range where the dependence is not as strong. These conditions also assume liquid phase and miscibility of species such that the particle is a homogeneous mixture.

\section{Summary and conclusions}

This paper presents the first results obtained from a two component aerosol study of miscible organic species using $\mathrm{CO}_{2}$ laser vaporization and VUV ionization in an ion trap mass spectrometer. The analysis of mixed particles, even laboratory generated two component aerosols, is a complicated process. Below we address the three questions posed in the Introduction.

Question 1: how does the fragmentation vary with $\mathrm{CO}_{2}$ laser energy and particle composition for one and two component systems? The degree of fragmentation observed in single particle mass spectra of a one component system was demonstrated to be a function of $\mathrm{CO}_{2}$ laser energy. In a two component system, the degree of fragmentation was shown to be a function of the particle composition. This observation indicates that the analysis of mixed particles may be far more complicated than anticipated for a two step process with soft vaporization if the degree of fragmentation can change as a function of composition. The mass spectra of the pure component cannot necessarily be used as a fingerprint for that same component in a mixture. The implications of this effect are also important for laboratory studies of particle reactions and subsequent product analysis, depending on the extent of particle composition change. In addition to showing that fragmentation is a function of $\mathrm{CO}_{2}$ energy and composition, we also showed that the key parameter that determines the extent of fragmentation, which is a proxy for the internal energy of the vaporized molecules, is the energy absorbed by the particle during desorption.

Question 2: how does the ionization delay profile vary with $\mathrm{CO}_{2}$ energy and composition for one and two component systems? The ionization delay profile in a one component system is shown to be strongly dependent on the vaporization energy. In a two component system, the delay profile is shown to strongly depend on the composition of the particle. The combined data suggest that the key parameter that governs the delay profile is also the energy absorbed by the particle during desorption. This finding has implications for potential field measurements. In the field, where each particle is not necessarily identical, it will be necessary to use a fixed ionization delay time (fixed time between the vaporization laser and ionization laser). This however could be problematic. For instance, if the instrument parameters are optimized for the maximum total ion signal with one species, these parameters may be far from ideal for a different species and will result in a sensitivity bias. Hanna et al. have shown delay profiles for 2,4-dihydroxybenzoic acid (DHB) and oleic acid at $15 \mathrm{~mJ}$ vaporization energy that varied widely; collecting data at the optimal delay time for oleic acid would result in almost no signal for DHB as its vaporization profile evolved much more quickly. ${ }^{8}$ Both one component and two component delay profiles in this study indicate this to be a valid concern. This effect may be diminished at high vaporization energies where there appears to be less of a composition dependence for pure particles, but this will come at the cost of increased fragmentation.

Question 3: for a two component system where the absorption crosssections are different, does the mass spectrum show a linear response with composition? No. The change in the degree of fragmentation with particle composition resulted in a non-linear dependence of ion signal on composition. This makes any attempt at quantification difficult. One possible way around this problem is to monitor all ion peaks associated with a species. But this also seems difficult to achieve in most cases, since organic species often have significant fragmentation even with VUV ionization, which can result in overlapping mass spectral peaks. For species that do not fragment as easily, or have similar absorption crosssections, quantitative analysis should be possible. Another possible way around this problem is to change the desorption method: impinging the particles on a heated surface would ensure that the desorption temperature is independent of the particle composition.

For atmospheric aerosols, the variation in the IR crosssection is not known. Many particles consist of inorganic species and a multitude of organic species. Studies on the IR crosssection of atmospheric aerosol particles (specifically for IR wavelengths relevant for a $\mathrm{CO}_{2}$ laser) would be interesting. Our studies have focused on a case where the crosssection changed by a factor of 4 . Whether or not this represents a "worst-case scenario" for field measurements remains to be determined.

Our system included a two step laser desorption/ionization system followed by an ion trap for mass analysis. Other two step laser desorption/ionization systems with a $\mathrm{CO}_{2}$ laser all use a TOF mass analyzer, which will result in less fragmentation than what is observed with an ion trap. ${ }^{8}$ One of the main 
differences between the TOF mass analyzer and an ion trap is the storage and measurement time for the ions. For a TOF mass analyzer the measurement/extraction time is on the order of microseconds. For an ion trap, the storage and measurement time is on the order of tens of milliseconds. As a result more fragmentation can occur in the ion trap experiments due to the longer time for unimolecular dissociation. Nevertheless the effects observed above should still be relevant for TOF instruments as long as some level of fragmentation is observed, which is the case for many types of analytes, particularly long chain hydrocarbons. ${ }^{9,25,30,31,33}$

\section{Appendix}

\section{Calculation of energy absorbed per particle during desorption}

Beer's law, which describes the absorption of light by a sample is given below, where $I$ is the intensity of light measured after the sample, $I_{\mathrm{O}}$ is the initial intensity of light, $\sigma$ is the absorption crosssection, $c$ is the concentration of the species, and $l$ is the path length the light travels:

$$
-\ln \left(\frac{I}{I_{\mathrm{o}}}\right)=\sigma c l
$$

In this case we are interested in the $\mathrm{CO}_{2}$ energy absorbed by the particle $\left(E_{\mathrm{abs}}\right)$. The energy measured after the particle is equal to the difference of the initial $\mathrm{CO}_{2}$ energy $\left(E_{\mathrm{i}}\right)$ and the energy absorbed by the particle $\left(E_{\mathrm{abs}}\right)$, thus the equation can be rewritten:

$$
-\ln \left(\frac{E_{\mathrm{i}}-E_{\mathrm{abs}}}{E_{\mathrm{i}}}\right)=\sigma c l
$$

Solving for the absorbed $\mathrm{CO}_{2}$ energy gives:

$$
E_{\mathrm{abs}}=E_{\mathrm{i}}(1-\exp (-\sigma c l))
$$

The initial $\mathrm{CO}_{2}$ energy the particle experiences is defined as the measured $\mathrm{CO}_{2}$ energy times the fraction of the $\mathrm{CO}_{2}$ beam intercepted by the particle:

$$
E_{\mathrm{i}}=E_{\mathrm{CO}_{2}} \frac{A_{\mathrm{p}}}{A_{\mathrm{CO}_{2}}}
$$

where $E_{\mathrm{CO}_{2}}$ is the measured $\mathrm{CO}_{2}$ energy, $A_{\mathrm{p}}$ is the area of the particle and $A_{\mathrm{CO}_{2}}$ is the area of the $\mathrm{CO}_{2}$ beam where it intersects the particle. The fraction of $A_{\mathrm{p}}$ over $A_{\mathrm{CO}_{2}}$ is included to take into account the geometric fraction of a flat beam profile intersecting the particle. We know the $\mathrm{CO}_{2}$ profile is Gaussian, so we could be off by as much as a factor of 2 in the absolute energy absorbed per particle, but the relative comparison between the pure and mixed particles will not be affected. Eqn (A4) can be rewritten as follows:

$$
E_{\mathrm{abs}}=E_{\mathrm{CO}_{2}} \frac{A_{\mathrm{p}}}{A_{\mathrm{CO}_{2}}}(1-\exp (-\sigma c l))
$$

The absorption crosssection $(\sigma)$ was measured with an FTIR spectrometer for pure components of oleyl alcohol or oleic acid and is given in units of $\mathrm{cm}^{2}$ molec ${ }^{-1}$. The concentration $c$ is given as the number density $\left(N_{\mathrm{p}}\right)$ of molecules in the particle (molecule per $\mathrm{cm}^{3}$ ). The path length $l$ (units of $\mathrm{cm}$ ) is approximated by calculating the height of a cylinder with the same volume and radius $\left(r_{\mathrm{p}}\right)$ as the particle studied which yields a path length of $4 r_{\mathrm{p}} / 3$. (For the purposes of this paper, the exact correction factor for path length is irrelevant since we are only comparing relative ratios).

Thus $E_{\text {abs }}$ can be determined as follows:

$$
E_{\mathrm{abs}}=E_{\mathrm{CO}_{2}} \frac{A_{\mathrm{p}}}{A_{\mathrm{CO}_{2}}}\left\{1-\exp \left[-\sigma N_{\mathrm{p}}\left(\frac{4 r_{\mathrm{p}}}{3}\right)\right]\right\}
$$

For the two component particle, eqn (A6) can be rewritten as follows where the contribution from each component is taken into account:

$$
E_{\mathrm{abs}}=E_{\mathrm{CO}_{2}} \frac{A_{\mathrm{p}}}{A_{\mathrm{CO}_{2}}}\left\{1-\exp \left[-\left(\sigma_{1} N_{1}+\sigma_{2} N_{2}\right) \frac{4 r_{\mathrm{p}}}{3}\right]\right\}
$$

$\sigma_{1}$ and $\sigma_{2}$ are the respective crosssections of each of the pure components and $N_{1}$ and $N_{2}$ are the molecular densities of each species in the mixed particle. There is some uncertainty in the absorption crosssection terms as absorption crosssections can vary with solvents and vary with temperature during the heating process. Also, the absorption crosssections determined for bulk solutions do not necessarily apply directly to aerosol particles.

\section{Acknowledgements}

This work was performed at the UBC Laboratory for Advanced Spectroscopy and Imaging Research (LASIR). The authors would like to thank the National Sciences and Engineering Research Council of Canada (NSERC), the Canadian Foundation for Climate and Atmospheric Sciences (CFCAS), the Canadian Foundation for Innovation (CFI), the British Columbia Knowledge Development Fund (BCKDF), the NRC-NSERC-BDC Nanotechnology Initiative, and the Canada Research Chair Program for financial support.

\section{References}

1 U. Pöschel, Angew. Chem., Int. Ed., 2005, 44, 7520-7540.

2 B. J. Finlayson-Pitts and J. N. Pitts Jr., Chemistry of the Upper and Lower Atmosphere: Theory, Experiments, and Applications, Academic Press, San Diego, 2000.

3 M. Kanakidou, J. H. Seinfeld, S. N. Pandis, I. Barnes, F. J. Dentener, M. C. Facchini, R. Van Dingenen, B. Ervens, A. Nenes, C. J. Nielsen, E. Swietlicki, J. P. Putaud, Y. Balkanski, S. Fuzzi, J. Horth, G. K. Moortgat, R. Winterhalter, C. E. L. Myhre, K. Tsigaridis, E. Vignati, E. G. Stephanou and J. Wilson, Atmos. Chem. Phys., 2005, 5, 1053-1123.

4 Y. Rudich, Chem. Rev., 2003, 103, 5097-5124.

5 J. Cabalo, A. Zelenyuk, T. Baer and R. E. Miller, Aerosol Sci. Technol., 2000, 33, 3-19.

6 A. Lazar, P. T. A. Reilly, W. B. Whitten and J. M. Ramsey, Environ. Sci. Technol., 1999, 33, 3993-4001.

7 M. R. Canagaratna, J. T. Jayne, J. L. Jimenez, J. D. Allan, M. R. Alfarra, Q. Zhang, T. B. Onasch, F. Drewnick, H. Coe, A. Middlebrook, A. Delia, L. R. Williams, A. M. Trimborn, M. J. Northway, P. F. DeCarlo, C. E. Kolb, P. Davidovits and D. R. Worsnop, Mass Spectrom. Rev., 2007, 26, 185-222.

8 S. J. Hanna, P. Campuzano-Jost, E. A. Simpson, I. Burak, M. W. Blades, J. H. Hepburn and A. K. Bertram, Phys. Chem. Chem. Phys., 2009, 11, 7963-7975.

9 M. J. Northway, J. T. Jayne, D. W. Toohey, M. R. Canagaratna, A. Trimborn, K. I. Akiyama, A. Shimono, J. L. Jimenez, P. F. DeCarlo, K. R. Wilson and D. R. Worsnop, Aerosol Sci. Technol., 2007, 41, 828-839. 
10 S. J. Hanna, P. Campuzano-Jost, E. A. Simpson, D. B. Robb, I. Burak, M. W. Blades, J. W. Hepburn and A. K. Bertram, Int. J. Mass Spectrom., 2009, 279, 134-146.

11 J. D. Hearn and G. D. Smith, J. Phys. Chem. A, 2004, 108, 10019-10029.

12 J. D. Hearn, A. J. Lovett and G. D. Smith, Phys. Chem. Chem. Phys., 2005, 7, 501-511.

13 J. D. Hearn and G. D. Smith, Int. J. Mass Spectrom., 2006, 258, 95-103.

14 D. Voisin, J. N. Smith, H. Sakurai, P. H. McMurry and F. L. Eisele, Aerosol Sci. Technol., 2003, 37, 471-475.

15 B. W. LaFranchi, J. Zahardis and G. A. Petrucci, Rapid Commun. Mass Spectrom., 2004, 18, 2517-2521.

16 B. W. LaFranchi and G. A. Petrucci, Int. J. Mass Spectrom., 2006, 258, 120-133.

17 J. Zahardis, B. W. LaFranchi and G. A. Petrucci, Atmos. Environ., 2006, 40, 1661-1670.

18 Y. X. Su, M. F. Sipin, H. Furutani and K. A. Prather, Anal. Chem., 2004, 76, 712-719.

19 A. Zelenyuk, J. Cabalo, T. Baer and R. E. Miller, Anal. Chem., 1999, 71, 1802-1808.

20 A. Zelenyuk and D. Imre, Aerosol Sci. Technol., 2005, 39, 554-568.

21 M. Bente, T. Adam, T. Ferge, S. Gallavardin, M. Sklorz, T. Streibel and R. Zimmermann, Int. J. Mass Spectrom., 2006, 258, 86-94.

22 M. Bente, M. Sklorz, T. Streibel and R. Zimmermann, Anal. Chem., 2009, 81, 2525-2536.

23 B. D. Morrical, D. P. Fergenson and K. A. Prather, J. Am. Soc. Mass Spectrom., 1998, 9, 1068-1073.

24 B. D. Morrical and R. Zenobi, Atmos. Environ., 2002, 36, 801-811.

25 J. N. Shu, S. K. Gao and Y. Li, Aerosol Sci. Technol., 2008, 42, $110-113$.
26 J. N. Shu, K. R. Wilson, M. Ahmed and S. R. Leone, Rev. Sci. Instrum., 2006, 77, 043106.

27 E. R. Mysak, K. R. Wilson, M. Jimenez-Cruz, M. Ahmed and T. Baer, Anal. Chem., 2005, 77, 5953-5960.

28 E. Woods, R. E. Miller and T. Baer, J. Phys. Chem. A, 2003, 107, 2119-2125.

29 E. Woods, G. D. Smith, Y. Dessiaterik, T. Baer and R. E. Miller, Anal. Chem., 2001, 73, 2317-2322.

30 E. Woods, G. D. Smith, R. E. Miller and T. Baer, Anal. Chem., 2002, 74, 1642-1649.

31 D. C. Sykes, E. Woods, G. D. Smith, T. Baer and R. E. Miller, Anal. Chem., 2002, 74, 2048-2052.

32 W. F. Rogge, L. M. Hildemann, M. A. Mazurek, G. R. Cass and B. R. T. Simonelt, Environ. Sci. Technol., 1991, 25, 1112-1125.

33 D. G. Nash, X. F. Liu, E. R. Mysak and T. Baer, Int. J. Mass Spectrom., 2005, 241, 89-97.

34 E. A. Simpson, P. Campuzano-Jost, S. J. Hanna, D. B. Robb, J. W. Hepburn, M. W. Blades and A. K. Bertram, Int. J. Mass Spectrom., 2009, 281, 140-149.

35 T. Ferge, F. Mühlberger and R. Zimmermann, Anal. Chem., 2005, 77, 4528-4538.

36 W. A. Harris, P. T. A. Reilly and W. B. Whitten, Int. J. Mass Spectrom., 2006, 258, 113-119.

37 T. Adam and R. Zimmermann, Anal. Bioanal. Chem., 2007, 389, 1941-1951.

38 B. Oktem, M. P. Tolocka and M. V. Johnston, Anal. Chem., 2004, 76, 253-261.

39 E. Gloaguen, E. R. Mysak, S. R. Leone, M. Ahmed and K. R. Wilson, Int. J. Mass Spectrom., 2006, 258, 74-85.

40 K. R. Wilson, M. Jimenez-Cruz, C. Nicolas, L. Belau, S. R. Leonet and M. Ahmed, J. Phys. Chem. A, 2006, 110, 2106-2113. 